\title{
2D Modeling of Solar Cell Radial Junction: Study of Carriers Charge Density and Photocurrent Density in Static Mode under Monochromatic Illumination
}

\author{
Moussa Ouédraogo', Raguilignaba Sam ${ }^{1,2}$, Alain Diasso² ${ }^{*}$, Bernard Zouma ${ }^{3}$, François Zougmoré2 \\ ${ }^{1}$ Laboratoire de Matériaux, d’Héliophysique et Environnement (LaMHE), Département de Physique, UFR/ST Université Nazi \\ Boni, Bobo Dioulasso, Burkina Faso \\ ${ }^{2}$ Laboratoire de Matériaux et Environnement, Département de Physique, UFR/SEA, Université Joseph Ki-Zerbo, Ouagadougou, \\ Burkina Faso \\ ${ }^{3}$ Laboratoire d'Energies Thermique Renouvelables (L.E.T.R.E), Université Joseph Ki-Zerbo, Ouagadougou, Burkina Faso \\ Email: mouedraogosh@gmail.com, *alinodiass@yahoo.fr
}

How to cite this paper: Ouédraogo, M., Sam, R., Diasso, A., Zouma, B. and Zougmoré, F. (2020) 2D Modeling of Solar Cell Radial Junction: Study of Carriers Charge Density and Photocurrent Density in Static Mode under Monochromatic Illumination. Energy and Power Engineering, 12, 568-577. https://doi.org/10.4236/epe.2020.1210035

Received: September 12, 2020

Accepted: October 24, 2020

Published: October 27, 2020

Copyright (๑) 2020 by author(s) and Scientific Research Publishing Inc. This work is licensed under the Creative Commons Attribution International License (CC BY 4.0).

http://creativecommons.org/licenses/by/4.0/

\begin{abstract}
A theoretical study of a polysilicon solar cell with a radial junction in static regime under monochromatic illumination is presented in this paper. The junction radial solar cell geometry is illustrated and described. The carriers' diffusion equation is established and solved under quasi-neutral base assumption with boundaries conditions and Bessel equations. New analytical expressions of electrons and holes density and photocurrent are found. The wavelength and structural parameters (base radius, emitter thickness) influences on charge carriers density and photocurrent are shown and examined.
\end{abstract}

\section{Keywords}

Electrons Density, Holes Density, Holes Photocurrent, Radial Junction

\section{Introduction}

Solar energy is an inexhaustible energy source. Solar cell technology has been pioneered in space industry, essentially because solar energy is one of the main power sources for satellites. However, space environment is a very harsh environment for electronic devices, such as silicon solar cells and other semiconductor based detectors. We can obtain energy by converting the solar energy in electrical energy with the semiconductor optoelectronic device, such as the vertical junction solar cell or planar junction [1]. Solar cell geometry seems to be 
very important for solar high performance. Especially since study conducted on cubic or cylindrical solar cell [2] showed a slight improvement in solar cell parameters in favor of a cylindrical model, notably the radial junction solar cell [3]. The originality of this work lies on its innovative and efficient aspect. The aim of this study is to investigate on charge carriers' density and photocurrent density respectively in the base and in the emitter of a radial junction polycrystalline solar cell.

\section{Materials and Methods}

In this study based on a $2 \mathrm{D}$ modeling of a polycrystalline silicon radial junction solar cell, we made the following hypothesis:

- The grains have identical structures and electrical properties. Therefore, the study is restricted to a grain whose model is schematized in Figure 1.

- The solar cell is subjected to monochromatic front side illumination;

- The electrical field of crystal lattice and the thickness of space charge region are neglected.

Figure 1 presents a radial junction solar cell geometry.

The operation of radial junction solar cell is based on optical absorption in vertical direction $(z)$. The photogenerated carriers' charge collection is made in radial direction.

\section{Study of Carriers' Charge Density}

The transfer phenomena of solar cell are model by the following equation of continuity numbered Equation (1) in cylindrical coordinates [4].

$$
\begin{aligned}
& \frac{\partial^{2} \delta(r, \theta, z)}{\partial r^{2}}+\frac{1}{r} \frac{\partial \delta(r, \theta, z)}{\partial r}+\frac{1}{r} \frac{\partial^{2} \delta(r, \theta, z)}{\partial \theta^{2}} \\
& +\frac{\partial^{2} \delta(r, \theta, z)}{\partial z^{2}}-\frac{\delta(r, \theta, z)}{L^{2}}=-\frac{g(z)}{D}
\end{aligned}
$$

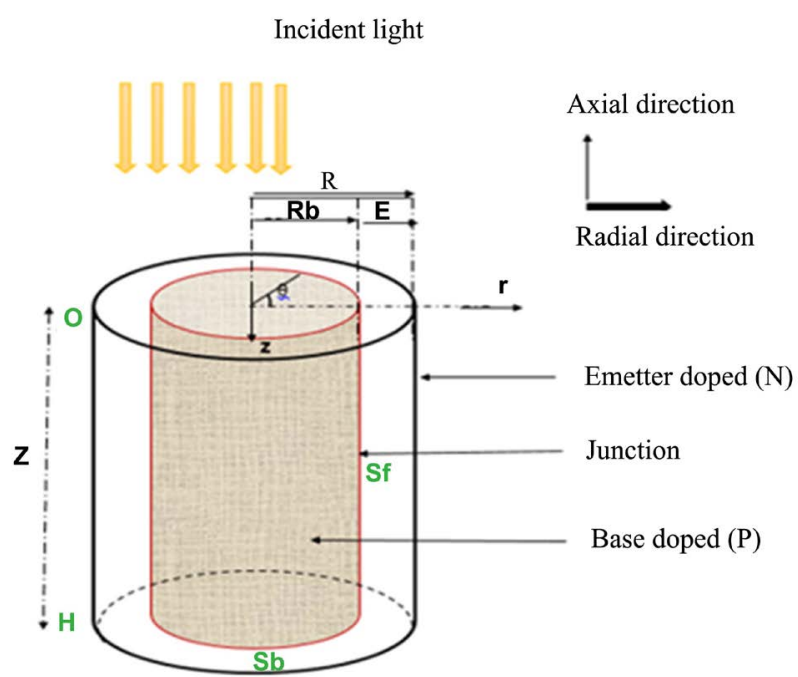

Figure 1. Radial junction model. 
where:

$g(z)$ is carriers generation rate [4] [5]:

$$
g=g(z)=\alpha(\lambda) \phi_{0}[1-R(\lambda)] \mathrm{e}^{-\alpha(\lambda) z}
$$

- $\delta(r, z):$ minority carriers' density

- $\quad D$ : carriers' diffusion coefficient

- $\quad L$ : carriers' diffusion length

As, we have an azimuthal symmetry, the angle $\theta$ is not processed [4] [5]. Therefore the continuity equation becomes:

- In the base region, the minority carriers' movement is governed by:

$$
\frac{\partial^{2} \delta_{n}(r, z)}{\partial r^{2}}+\frac{1}{r} \frac{\partial \delta_{n}(r, z)}{\partial r}+\frac{\partial^{2} \delta_{n}(r, z)}{\partial z^{2}}-\frac{\delta_{n}(r, z)}{L_{n}^{2}}=-\frac{g(z)}{D_{n}}
$$

- In the emitter region, the transport equation is given by:

$$
\frac{\partial^{2} \delta_{p}(r, z)}{\partial r^{2}}+\frac{1}{r} \frac{\partial \delta_{p}(r, z)}{\partial r}+\frac{\partial^{2} \delta_{p}(r, z)}{\partial z^{2}}-\frac{\delta_{p}(r, z)}{L_{p}^{2}}=-\frac{g(z)}{D_{p}}
$$

\section{1) Electrons density and photocurrent in the base}

\section{a) Electrons density expression}

Referring to Figure 1, the boundaries conditions are:

- At the junction $\left(r=R_{b}\right)$ :

$$
\left.\frac{\partial \delta_{n}(r, z)}{\partial r}\right|_{r=R_{b}}=-\frac{S_{f}}{2 D_{n}} \delta\left(r=R_{b}, z, \lambda\right)
$$

- At the rear side $(z=H)$ :

$$
\left.\frac{\partial \delta_{n}(r, z)}{\partial z}\right|_{z=H}=-\frac{S_{b}}{D_{n}} \delta(r, z=H, \lambda)
$$

A solution of equation is give by:

$$
\delta_{n}(r, z)=\sum_{k \geq 0}^{\infty} f(r, \lambda) \cos \left(c_{k} z\right)
$$

The eigenvalues $c_{k}$ which satisfy the boundary condition (6) are expressed by:

$$
c_{k} \tan \left(c_{k} H\right)=\frac{\delta_{b}}{D_{n}}
$$

Replacing the Equation (7) in Equation (5), the function $f(r, \lambda)$ can get the form:

$$
f(r, \lambda)=A_{k}(\lambda) I_{0}\left(\frac{r}{L_{n k}}\right)+M_{k}(\lambda)
$$

where the following expressions gives Equation (10):

$$
\begin{aligned}
M_{k}(\lambda)= & \frac{2 g_{0}(\lambda)}{D_{n}} \frac{c_{k} L_{n k}^{2}}{c_{k}^{2}+\alpha^{2}(\lambda)} \\
& \times \frac{\left[c_{k} \sin \left(c_{k} H\right)-\alpha(\lambda) \cos \left(c_{k} H\right)\right] \exp (-\alpha(\lambda) H)+\alpha(\lambda)}{c_{k} H+\sin \left(c_{k} H\right) \cos \left(c_{k} H\right)}
\end{aligned}
$$


Coefficients $A_{k}(\lambda)$ is obtained by boundary condition (5):

$$
A_{k}(\lambda)=-\frac{M_{k}(\lambda)}{\left(\frac{2 D_{n}}{L_{n k} S_{f}}\right) I_{0}^{\prime}\left(\frac{R_{b}}{L_{n k}}\right)+I_{0}\left(\frac{R_{b}}{L_{n k}}\right)}
$$

$I_{0}(x)$ is the modified Bessel function of the first kind of zero order and $I_{0}^{\prime}(x)$ is its first derivative with respect to its argument [6].

b) Electrons photocurrent density expression

The equation of photocurrent density is given by:

$$
J_{p h}^{b}=-\left.q D_{n} \int_{0}^{H} \frac{\partial \delta_{n}(r, z)}{\partial r}\right|_{r=R_{b}} \mathrm{~d} z
$$

Substituting $\delta_{n}(r, z)$ in Equation (12); this equation becomes:

$$
J_{p h}^{b}=q \frac{S_{f}}{2 c_{k}} \sum_{k \geq 0}^{\infty}\left(A_{k}(\lambda) I_{0}\left(\frac{R_{b}}{L_{n k}}\right)+M_{k}(\lambda)\right) \sin \left(c_{k} H\right)
$$

\section{2) Holes density and photocurrent in the emitter}

\section{a) Holes density expression}

Referring to Figure 1, the boundary condition in the emitter is:

- At the junction:

$$
\left.\frac{\partial \delta_{P}}{\partial r}\right|_{r=E}=-\frac{S_{f}}{2 D_{p}} \delta_{P}\left(r=R_{b}, z\right)
$$

- At the rear side:

$$
\left.\frac{\partial \delta_{P}}{\partial r}\right|_{z=H}=-\frac{S_{P}}{D_{p}} \delta_{P}(r, z=H)
$$

- The continuity Equation (4) general solution is:

$$
\delta_{p}(r, z)=\sum_{k \geq 0}^{\infty} f_{p}(r, \lambda) \cos \left(a_{k} z\right)
$$

The eigenvalues $a_{k}$ which satisfy the boundary condition Equation (15) are given by:

$$
a_{k} \tan \left(a_{k} H\right)=-\frac{S_{P}}{D_{P}}
$$

The function $f_{p}(r, \lambda)$ can be shown to have the form:

$$
f_{p}(r, \lambda)=B_{k}(\lambda) J_{0}\left(\frac{r}{L_{p k}}\right)+N_{K}(\lambda)
$$

$J_{0}(x)$ is the modified Bessel function of the first kind of zero order and $J_{0}^{\prime}(x)$ is its first derivative with respect to its argument.

\section{b) Holes photocurrent density expression}

The photocurrent density is given by:

$$
J_{p h}^{E}=\left.q D_{p} \int_{0}^{H} \frac{\partial \delta_{p}(r, z, \lambda)}{\partial r}\right|_{r=R_{b}} \mathrm{~d} z
$$


After calculation of Equation (19); this equation can be rewritten:

$$
J_{p h}^{E}=q D_{p} \int_{0}^{H}\left(-\frac{S_{f}}{2 D_{p}} \sum_{k \geq 0}^{\infty} f_{P}(E, \lambda) \cos \left(a_{k} z\right)\right) \mathrm{d} z
$$

Solar cell photocurrent is then obtained by adding the above two conditions:

$$
J_{p h}=J_{p h}^{b}+J_{p h}^{E}
$$

So we can write this following Equation (22)

$$
J_{p h}=-\left.q D_{n} \int_{0}^{H} \frac{\partial \delta_{n}(r, z, \lambda)}{\partial r}\right|_{r=R_{b}} \mathrm{~d} z+\left(\left.q D_{p} \int_{0}^{H} \frac{\partial \delta_{p}(r, z, \lambda)}{\partial r}\right|_{r=E} \mathrm{~d} z\right)
$$

\section{Results and Discussions}

We present here the simulation results obtained from the previous modeling equations by using Mathcad software.

\subsection{Electrons Density and Photocurrent in the Base}

The effects of wavelength and base radius on electrons density and photocurrent density are presented and analyzed.

\subsubsection{Radius $\boldsymbol{R}_{b}$ Effects on Electrons Density}

In Figure 2, we present the variations of electrons density versus base radius for different values of base depth.

We observe that the electrons density and base radius of solar cell vary in the same order. The generation of electrons in the base is important because base radius increase. Also electrons density decrease versus base depth.

\subsubsection{Wavelength Effects on Electrons Density}

In Figure 3, we present the variations of electrons density versus wavelength for different values of solar cell thickness.



Figure 2. Electrons density profile versus radius. $\lambda=800 \mathrm{~nm}, R_{b}=150 \mu \mathrm{m}, S_{f}=10^{4} \mathrm{~cm} / \mathrm{s}$, $S b=10^{3} \mathrm{~cm} / \mathrm{s}, H=300 \mu \mathrm{m}, L_{n}=100 \mu \mathrm{m}$. 


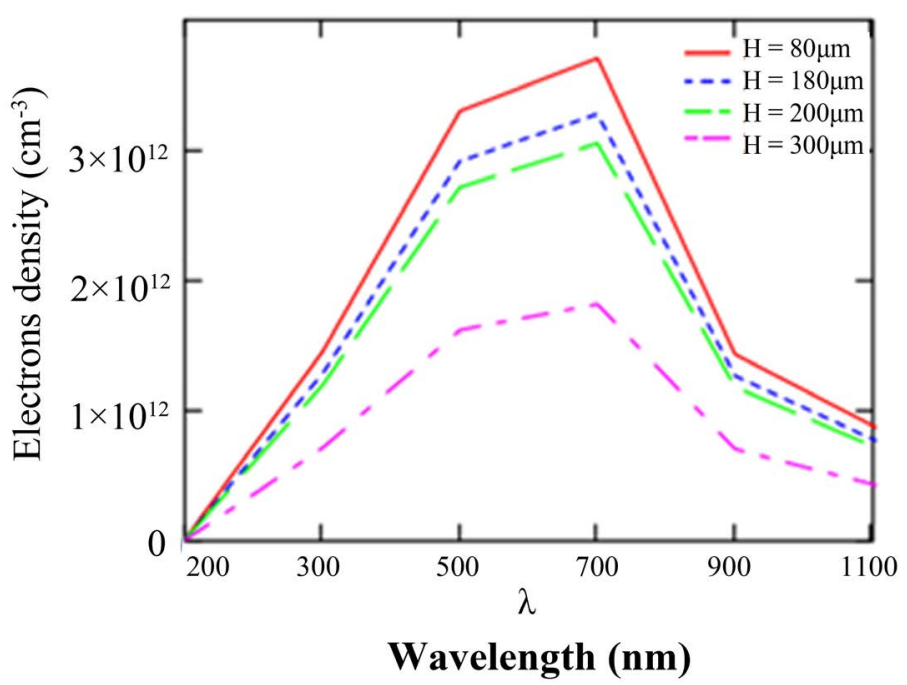

Figure 3. Profile of the density of electrons in the base as a function of length wave $\lambda$ for different values of the thickness $H$ of the solar cell. $R_{b}=150, S_{f}=10^{4} \mathrm{~cm} / \mathrm{s}, S_{b}=10^{3} \mathrm{~cm} / \mathrm{s}$, $L_{n}=100 \mu \mathrm{m}$.

Figure 3 shows low values of electrons density for low wavelength and big wavelength. The variations due to big values are explained by silicon energy gap $(1.12 \mathrm{eV})$. Also, the weak absorption for low and big values explain these electrons density variations. The absorption peak is observed around $700 \mathrm{~nm}$.

\subsubsection{Wavelength Effects on Photocurrent Density}

Figure 4 shows the profile of photocurrent density versus wavelength for various values of junction recombination velocity Sf.

We remark a low photocurrent density for low and big wavelength. The absorption peak is notified around $700 \mathrm{~nm}$.

These phenomena are explained by electrons density variations. In additional, photocurrent density increase for $10^{2} \mathrm{~cm} / \mathrm{s} \leq S_{f} \leq 10^{6} \mathrm{~cm} / \mathrm{s}$.

Because more electrons cross the junction. For $S_{f} \prec 10^{2} \mathrm{~cm} / \mathrm{s}$.

Photocurrent density is almost zero because there is a low displacement and strong accumulation of electrons near the junction. Solar cell is then an open circuit state [1] [7].

\subsection{Study of Holes Density and Holes Photocurrent Density in the Emitter}

The following figure presents variations of holes density as a function of emitter thickness for different values of the depth creating more electrons because the emitter thickness increases.

\subsubsection{Emitter Thickness Effects on Holes Photocurrent Density}

In Figure 5, below we study the evolution of photocurrent density versus holes recombination velocity

It is observed that the holes density increase with the emitter thickness increase. This behavior is explained by the increase of incident light absorption 


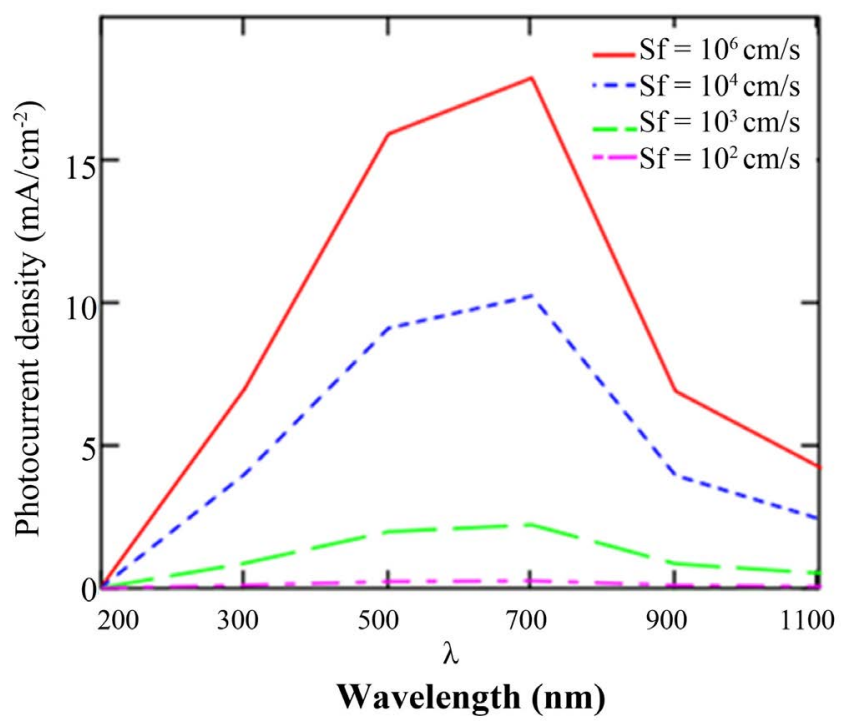

Figure 4. Profile of the photocurrent density as a function of the wavelength for different values of the recombination speed of the radius $R_{b}$ of the base with, $R_{b}=150 \mu \mathrm{m}, S_{b}=10^{4}$ $\mathrm{cm} / \mathrm{s}, H=300 \mu \mathrm{m}, L_{n}=120 \mu \mathrm{m}$.

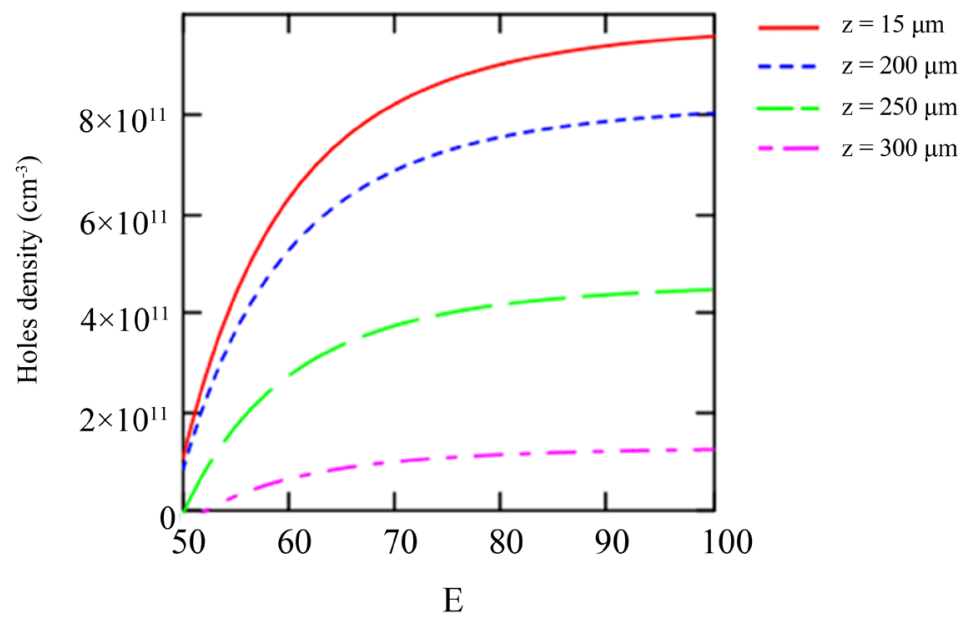

Emitter thickness $(\mu \mathrm{m})$

Figure 5. Profile of the holes density in the emitter as a function of the thickness of the transmitter for different values of the length of the depths $z$ with: $R_{b}=150 \mu \mathrm{m}, S f=10^{4}$ $\mathrm{cm} / \mathrm{s}, S b=10^{3} \mathrm{~cm} / \mathrm{s}, H=300 \mu \mathrm{m}, L_{n}=100 \mu \mathrm{m}$.

due to the emitter thickness increase. Also, the holes density decrease with the base depth the holes photogeneration decrease.

\subsubsection{Emitter Thickness Effects on Holes Photocurrent Density}

In Figure 6, the variations of holes photocurrent density versus holes recombination velocity for various values of emitter thickness.

The increase of holes photocurrent density is observed with the emitter thickness increase. This is due to the fact that more the emitter thickness increases, more there is a large absorption of incident photons. These is consequently generates more carriers in the emitter region. 


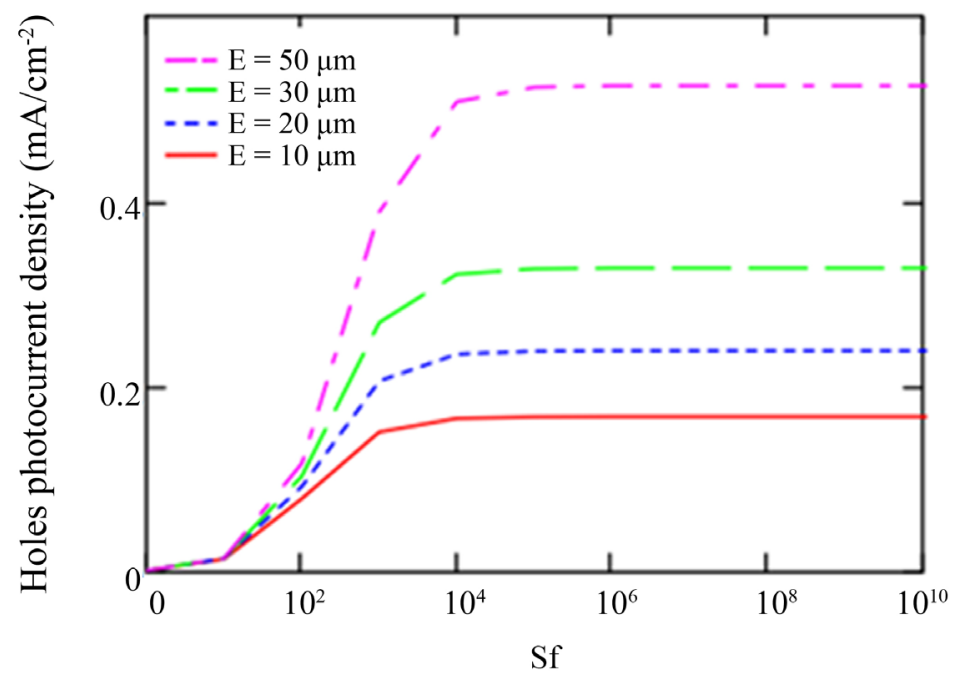

Holes recombination velocity $\left(\mathrm{cm} \cdot \mathrm{s}^{-1}\right)$

Figure 6. Profile of the photocurrent density as a function of the speed of recombination of holes at the junction for different values of the thickness of the transmitter with: $R_{b}=$ $150, S_{b}=10^{3} \mathrm{~cm} / \mathrm{s}, H=300 \mu \mathrm{m}, L_{n}=120 \mu \mathrm{m}$.

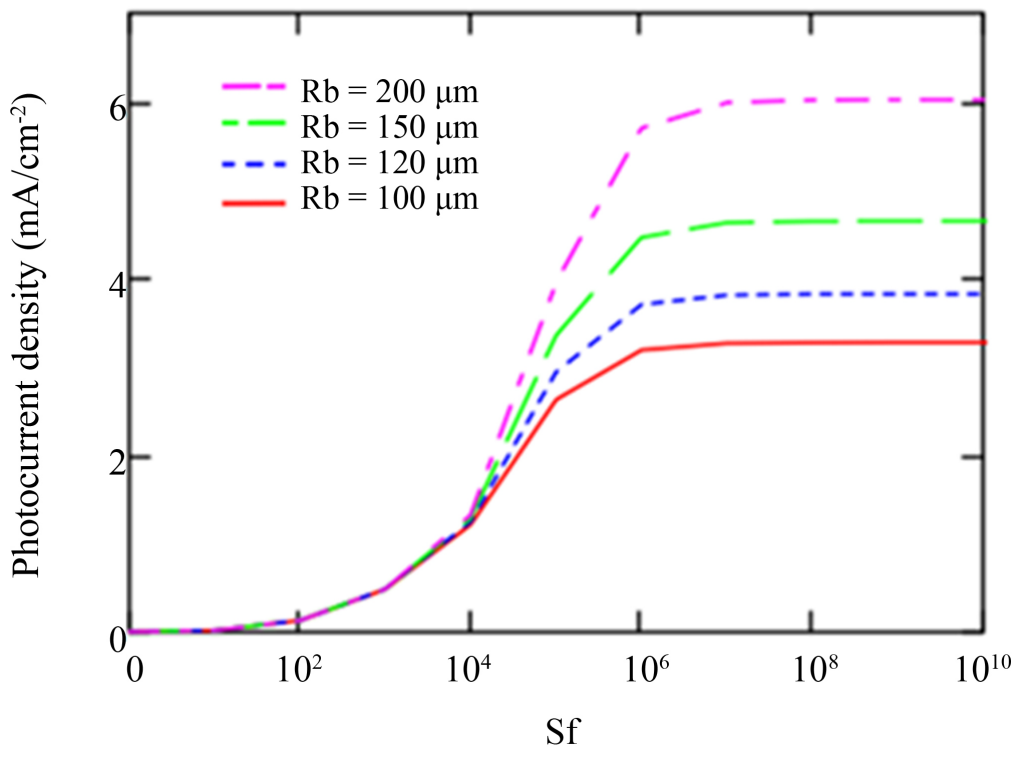

charges carriers' density recombination velocity $(\mathrm{cm} / \mathrm{s})$

Figure 7. Profile of the photocurrent density as a function of the recombination rate of holes at the junction for different values of the base radius with: $S_{b}=10^{3} \mathrm{~cm} / \mathrm{s}, H=300$ $\mu \mathrm{m}, L_{n}=120 \mu \mathrm{m}$.

\subsubsection{Solar Cell Photocurrent Density versus Base Radius and Emitter Thickness}

The photocurrent density of solar cell is linked to the electrons and holes recombination governed respectively by electrons recombination velocity and holes recombination velocity.

In Figure 7, the profile of photocurrent density as a function of dynamic velocity to the junction for various values of base radius $R_{b}$. 


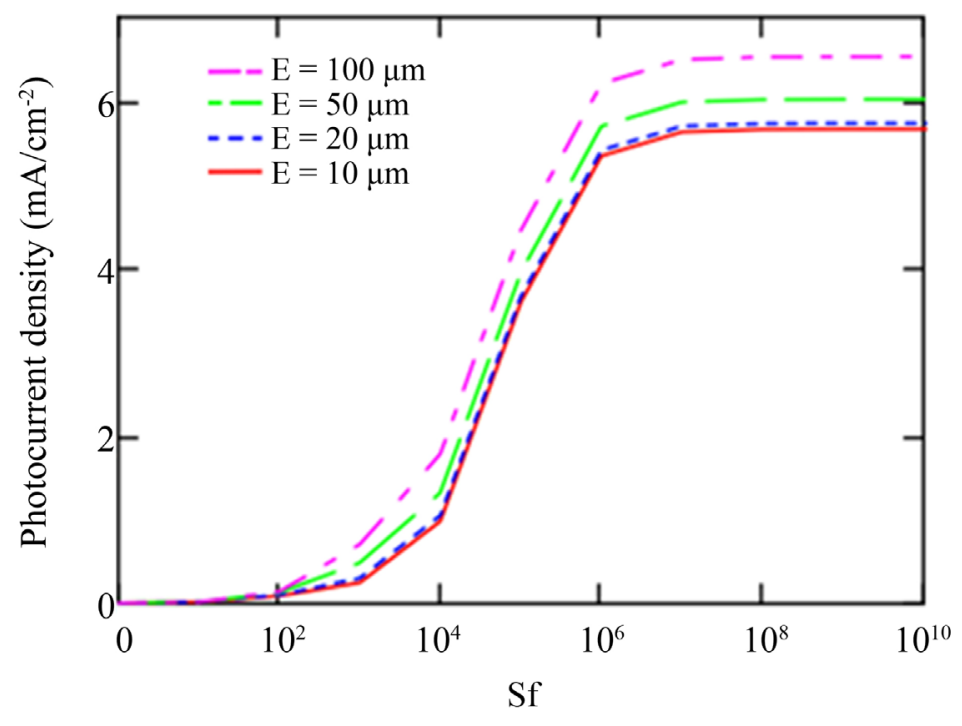

charges carriers' density recombination velocity $(\mathrm{cm} / \mathrm{s})$

Figure 8. Profile of the photocurrent density as a function of the speed of recombination of holes at the junction for different values of the thickness of the transmitter with: $R_{b}=$ $150 \mu \mathrm{m}, S_{b}=10^{3} \mathrm{~cm} / \mathrm{s}, H=300 \mu \mathrm{m}, L_{n}=120 \mu \mathrm{m}$.

The photocurrent increases with the increase of base radius $R_{b}$. The base radius increase creates a large incident light absorption, thus leading to a large carrier's photogeneration in solar cell.

In Figure 8, we present the photocurrent density curves as a function of recombination velocity for different values of emitter thickness.

The photocurrent density increases versus carriers' recombination velocity to the junction and the emitter thickness. These variations are explained on previous lines.

These phenomena are explained by charge carriers' density variations. Also the charge carriers' photocurrent density increase for $10^{2} \mathrm{~cm} / \mathrm{s} \leq S_{f} \leq 10^{6} \mathrm{~cm} / \mathrm{s}$ because more charge carriers cross the junction.

For $S_{f} \prec 10^{2} \mathrm{~cm} / \mathrm{s}$, photocurrent density is almost zero because there is a low displacement and strong storage of charge carriers near the junction. So the solar cell is an open circuit state [2] [7] and for $S_{f} \geq 10^{6} \mathrm{~cm} / \mathrm{s}$ the solar cell is a short circuit state

\section{Conclusions}

This paper carried out 2D modelling of radial junction of solar cell. The effects of base radius, emitter thickness and wavelength on carriers' charge density and carriers' photocurrent density are investigated.

Also, the influences of base radius, emitter thickness and charges carriers' recombination velocity on photocurrent density of solar cell are presented and analyzed. The results of this study which are in good agreement with others research works [3] validate thus ours results.

The 2D modeling of solar cell radial junction is studied in first time in cylin- 
drical coordinates according our research. So this work will permit to study better the radial junction of solar cell. In the future, we will try to do an experimental study to confirm theoretical results with experimental results.

\section{Conflicts of Interest}

The authors declare no conflicts of interest regarding the publication of this paper.

\section{References}

[1] Chossat, M. and Privat, Y. (2001) Mathématiques de l'ingénieur Dunod. 4e Edition.

[2] Samb, M.L., Zoungrana, M., Sam, R., Dione, M.M., Deme, M.M. and Sissoko, G. (2010) Etude en modélisation 3D d'une photopile au silicium régime statique placée dans un champ magnétique et sous éclairement multispectral: Détermination des paramètres électriques. Journal des Sciences, No. 4, 23-38.

[3] Trabelsi, A., Zouri, A. and Ben Arab, A. (2009) Modeling of Polycrystalline N+/P Junction Solar Cell with Columnar Cylindrical Grain. Revue des Energies Renouvelables, 12, 279-297.

[4] Kayes, B.M. and Atwater, H.A. (2005) Comparison of the Device Physics Principals of Planar and Radial p-n Junction Nanorod Solar Cells. Journal of Applied Physics, 97, 114302-114311. https://doi.org/10.1063/1.1901835

[5] Elnahwy, S. and Adeeb, N. (1988) Exact Analysis of a Three Dimension Cylindrical Model for a Polycrystalline Solar Cell. Journal of Applied Physics, 64, 5214. https://doi.org/10.1063/1.342435

[6] Leye, S.N., Diouf, A., Mbodji, S. and Sissoko, G.G. (2017) A Method to Determine the Transient Capacitance of the Bifacial Solar Cell Considering the Cylindrica Grain and the Dynamic Junction Velocity (Sf). EAI Endorsed Transactions on Collaborative Computing, 3, e5. https://doi.org/10.1063/1.342435

[7] Zouma, B., Maiga, A.S., Dieng, M., Zougmore, F. and Sissoko, G. (2009) 3D Approach of Spectral Response for a Bifacial Silicon Solar Cell under a Constant Magnetic Field. Global Journal of Pure and Applied Sciences, 15, 117-124.

https://doi.org/10.4314/gjpas.v15i1.44908 